\title{
An area efficient memory-less ROM design architecture for direct digital frequency synthesizer
}

\author{
Salah Alkurwy' ${ }^{1}$, Sawal H. Ali ${ }^{2}$, Md. Shabiul Islam³ ${ }^{3}$ Faizul Idros ${ }^{4}$ \\ ${ }^{1}$ College of Engineering, University of Diyala, Iraq \\ ${ }^{2}$ Faculty of Engineering, Universiti Kebangsaan Malaysia (UKM), Malaysia \\ ${ }^{3}$ Multimedia University (MMU), Malaysia \\ ${ }^{4}$ Faculty of Engineering, Universiti Teknologi MARA (UiTM), Malaysia
}

\begin{tabular}{l} 
Article Info \\
\hline Article history: \\
Received Jan 6, 2020 \\
Revised Jul 3, 2020 \\
Accepted Aug 5, 2020 \\
\hline Keywords: \\
Direct digital frequency syn- \\
thesizer (DDFS) \\
Kogge-stone (KG) adder \\
Memory-less ROM \\
Read only memory (ROM)
\end{tabular}

\begin{abstract}
This paper introduces a new technique of designing a read-only memory (ROM) circuit, namely; memory-less ROM as a novel approach to designing the ROM lookup table (LUT) circuit for use in a direct digital frequency synthesizer (DDFS). The proposed DDFS design uses the pipelined phase accumulator (PA) based on the kogge-stone (KS) adder. Verilog HDL programming is encoded on the architecture circuit of pipelined PA and contrasted with other PA based on various adders. The obtained results define the KS adder as having good capabilities for improving the throughput. In addition to the quarter symmetry technique, the built memory-less ROM to obtain the quarter sine amplitude waveform is proposed and implemented in the DDFS system. The implementation of the proposed technique replaces the necessary ROM registers (384 D flip-flops) and multiplexers with simple logic gate circuits instead of traditional ROMs. This technique would reduce the area size and cell count by $56 \%$ and $32.6 \%$ respectively.
\end{abstract}

This is an open access article under the $\underline{C C B Y-S A}$ license.

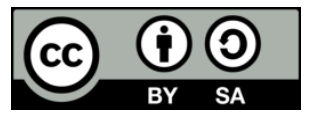

\section{Corresponding Author:}

Salah Alkurwy,

College of Engineering,

University of Diyala,

32001 Baqubah, Diyala, Iraq.

Email: salahalkurwy@uodiyala.edu.iq

\section{INTRODUCTION}

The PA contributes to the speed performance of the DDFS. The PA speed performance is attributable to the pipelining technique and fast adders [1], therefore, improving these features will increase the PA operating frequency. Reducing the read-only memory (ROM) size and decreasing the power consumption are the common goals of research. The conventional ROM size requires a $\left(2^{\mathrm{P}} \times \mathrm{P}\right)$, where $\mathrm{P}$ represents the addressing phase bits. The ROM size exponentially increases with the number of addressing phase bits. Quarter wave symmetry technique was applied in [2] and [3] to reduce $75 \%$ of the ROM size, by addressing the ROM with only quarter sine wave by P-2 phase bit, and to utilize the two highest, most significant bits (MSBs) for accomplishing the half and full ( $\pi$ and $2 \pi$ ) sine waveforms with 1 'complement and 2'complement, respectively. Quad line approximation technique as another technique [4], to design a ROM circuit with a relatively small ROM bit size. An adder, multiplexer, and complementor were needed as additional hardware to convert the amplitude phase into a sine amplitude waveform. In addition to the mentioned ROM design approaches, several techniques such the ROM-DAC and ROM-less techniques were applied in [5-7] respectively, to architect the DDFS system. 
The current paper presents a high-speed DDFS with pipelining PA based on clock shifting technique. In the proposed work, memory-less ROM is implemented as a novel phase-to-amplitude converter (PAC) method. PA is a generator that provides a digital phase with $0-2 \pi$ range values. The PA architecture consists of the adder and register, as shown in Figure 1.

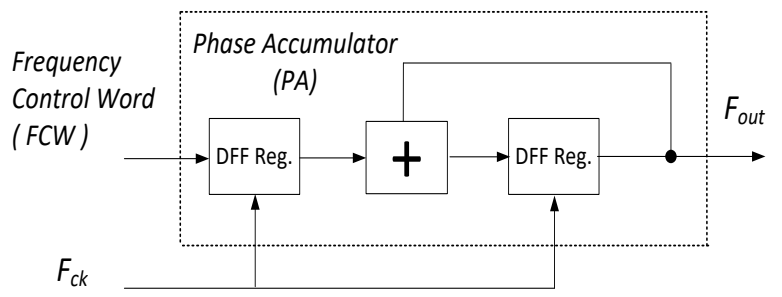

Figure 1. Phase accumulator architecture

Kogge-stone adder (KS adder) is a parallel prefix adder family that consists of groups of $p$ and $g$ cells. The arithmetic operation for a KS adder is as follows:

$$
p_{i}=x_{i} \oplus y_{i}, g_{i}=x_{i} \cdot y_{i}
$$

In (2), (3), and (4) presents the sum and carry out of a 4-bit KS adder.

$$
\begin{aligned}
& S_{0}=p_{0} \oplus c_{i n .} \\
& S_{2: 1}=p_{2: 1} \oplus C_{2: 1} \\
& C_{\text {out }}=\left(g_{3}+p_{3} \cdot g_{2}\right)+p_{3} \cdot p_{2} C_{2}
\end{aligned}
$$

The block circuit diagram of the proposed 4-bit KS adder in Figure 2 shows the groups of $p$ and $g$ cells. These cells connect with each other to achieve the summation outputs of the parallel prefix tree with minimum delay.

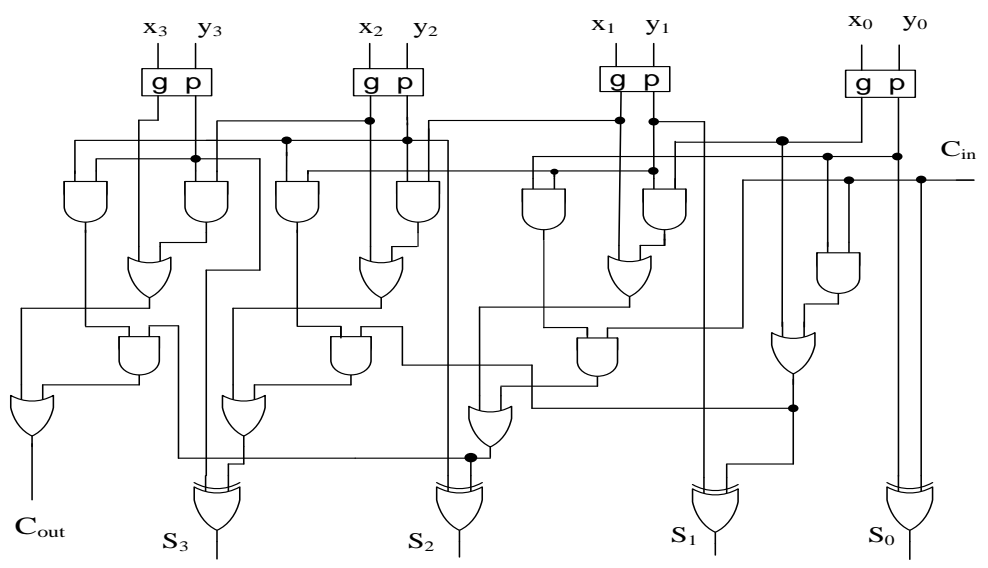

Figure 2. Block circuit diagram of 4-bit KS adder

Pipelining is a technique in which the given PA is partitioned into a number of subphase accumulators that should be sequentially performed. As a result, the frequency operation speed is multiplied (doubled) as much as the partitioned pipelining stage registers. Parallel prefix adders are fast adder circuits that can be used for PA binary addition. Parallel prefix adders, such as Sklansky [8], KS [9-12], Brent-Kung (BK) [13], Beaumont-smith (BS) [14] as well the carry look-ahead (CLA) [15] and ripple carry adder (RCA) [16] are applied in the conventional 12-bit pipelined PA design, as shown in Figure 3. The PAs' 
designs are coded in Verilog hardware description language (HDL) code and verified with Cyclone III FPGA kit and Quartus II software. The frequency speed comparison result shown in Figure 4 demonstrates that the KS-based pipelined PA outperforms the other PA architectures.

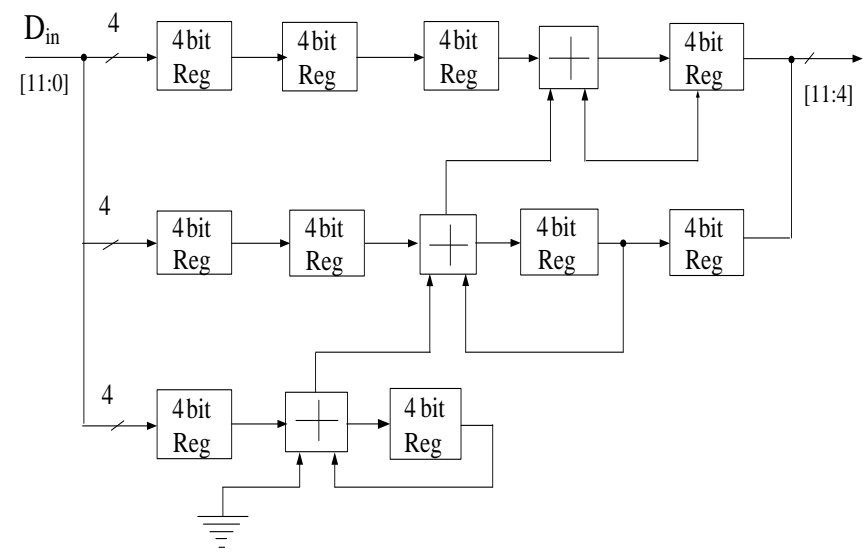

Figure 3. Pipelined PA design circuit diagram

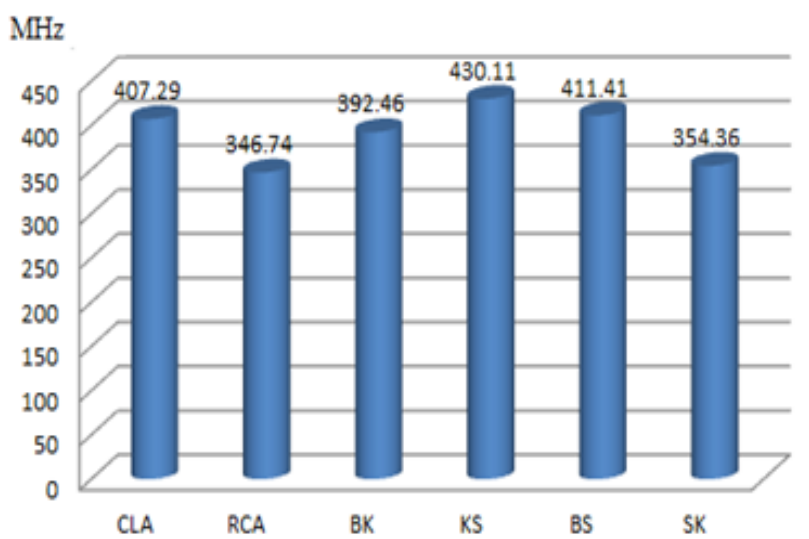

Figure 4. Frequency operating speed comparison of KS-based pipelined PA architecture and other PA architectures

\section{PHASE TO AMPLITUDE CONVERTER (PAC)}

PAC is a storage memory of the addressing data in the DDFS system, and it is used to provide the required digital sine amplitude waveform for representing the PA output. The ROM size area exponentially increases with number of addressing input bits. Moreover, 75\% of the ROM size can be saved using the quarter symmetry technique in this design, which can be achieved by addressing the ROM with only quarter sine wave by P-2 phase bit. The two highest MSBs are utilized to achieve the half $(\pi)$ and full sine $(2 \pi)$ waveforms with the 1 'complement and 2'complement, respectively. The 2'complement is achieved by adding a full adder at the output gate to accomplish the full sine wave. Adding half of the LSB offset to the stored memory address of the display values eliminates the 2'complement full adder hardware from the proposed design and replaces them with only 1 'cmplement.

The phase mapping address values are mathematically calculated based on the following equation:

$$
\sin A=\left(2^{A}-1\right) \times \sin \left(\frac{\pi}{2} \times \frac{\left[0:\left(2^{A}-1\right)\right]}{2^{A}}\right)
$$

where $\mathrm{A}$ is the number of the addressing phase bits of the ROM input. The achieved fraction values $(0-0.99)$ of $\sin A(5)$ are converted using MATLAB into 6-bit binary numbers (0-63) to collect the memory-less ROM circuit based on BCD-to-7-segment display technique. 


\section{MEMORY-LESS DESIGN ARCHITECTURE}

The basic concept of BCD-to-7-segment decoder is explained as follows: BCD is used to provide the (0-9) numbers listed in the binary digits' rows, whereas the listed column lines based on the binary digits' rows is used to provide the segment display. The memory-less ROM replacement circuit is designed on the basis of the 7-segment display and the technique applied in [17-19]. The suggested quarter sinewave values, which are listed in 6-bit binary digits in rows $0-63\left(\mathrm{X}_{5}: \mathrm{X}_{0}\right)$, are used as a counter of the memory-less ROM circuit. The required ROM output bits are listed in the column line $\left(A_{5}: A_{0}\right)$ on the basis of the binary digit rows as showon in Figure 5.

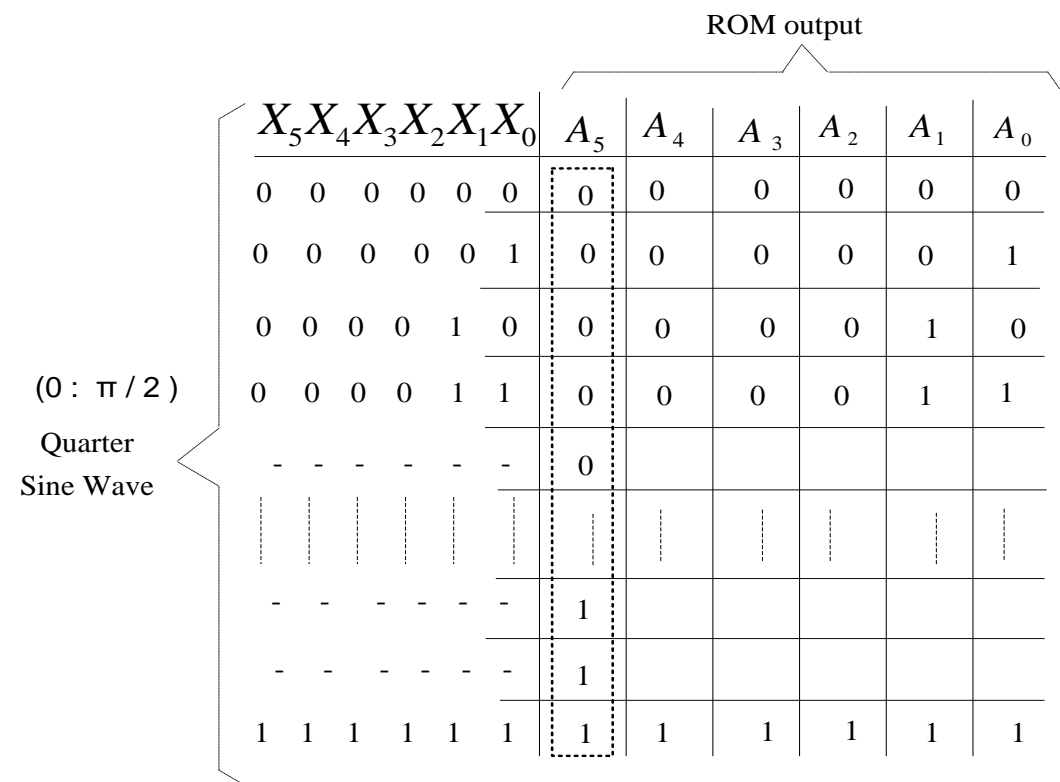

Figure 5. Memory-less design concept

Karnaugh map and Boolean expression were used to simplify the $\left(\mathrm{X}_{5}: \mathrm{X}_{0}\right)$ created logic circuits. On this basis, the $\sin A$ equation for $\mathrm{A}_{5}$ and $\mathrm{A}_{4}$ are expressed as follows:

$$
\begin{aligned}
& A_{5}=X_{0}+\overline{X_{0}} X_{1}\left(X_{2}+X_{3} X_{4}\right) \\
& A_{4}=X_{0}\left(X_{3}+X_{2}+X_{1}\right)+\overline{X_{0}} X_{1} \overline{X_{2}}\left(\overline{X_{3}}+X_{3} \overline{X_{4}}\right)+\overline{X_{0}} X_{2}\left(X_{3}+\overline{X_{3}} X_{4} X_{5}\right)
\end{aligned}
$$

and so on for the rest of the equations $\left(\mathrm{A}_{3}, \mathrm{~A}_{2}, \mathrm{~A}_{1}\right.$ and $\left.\mathrm{A}_{0}\right)$.

The existence of ROM look-up-table in the DDFS system is necessary to convert the phase signal into an amplitude sine waveform. To achieve the desired values of the ROM circuit, it must involve a number of registers and multiplexers equal to the number of required points of the amplitude sine waveform. Based on the Karnaugh map, the equations of the obtained values are simplified and presented in basic AND, OR, and XOR logic gates. The conventional 6-bit ROM requires 64 6-bit registers (384 D flip-flops (DFFs)) and six multiplexers $(64 \times 1)$. Applying the memory-less ROM circuit in the proposed design as shown in Figure 6 replaces 139 of AND, OR, and XOR logic circuits instead of the 384 DFF registers and multiplexers. Table 1 lists the comparison results between the conventional ROM LUT and the proposed memory-less ROM circuit.

Table 1. Comparison results of conventional and memory-less ROM logic gates

\begin{tabular}{llll}
\hline (6-bit) ROM & Register $/$ bit & Multiplexer $/$ bit & Logic gates \\
\hline Conventional & $64 \times(6-$ bit $)=384$ & $6 \times(64 \times 1)+(6 \times 1)=390$ & $1536+780=2316$ \\
Memory-less & --- & --- & 139 \\
\hline
\end{tabular}




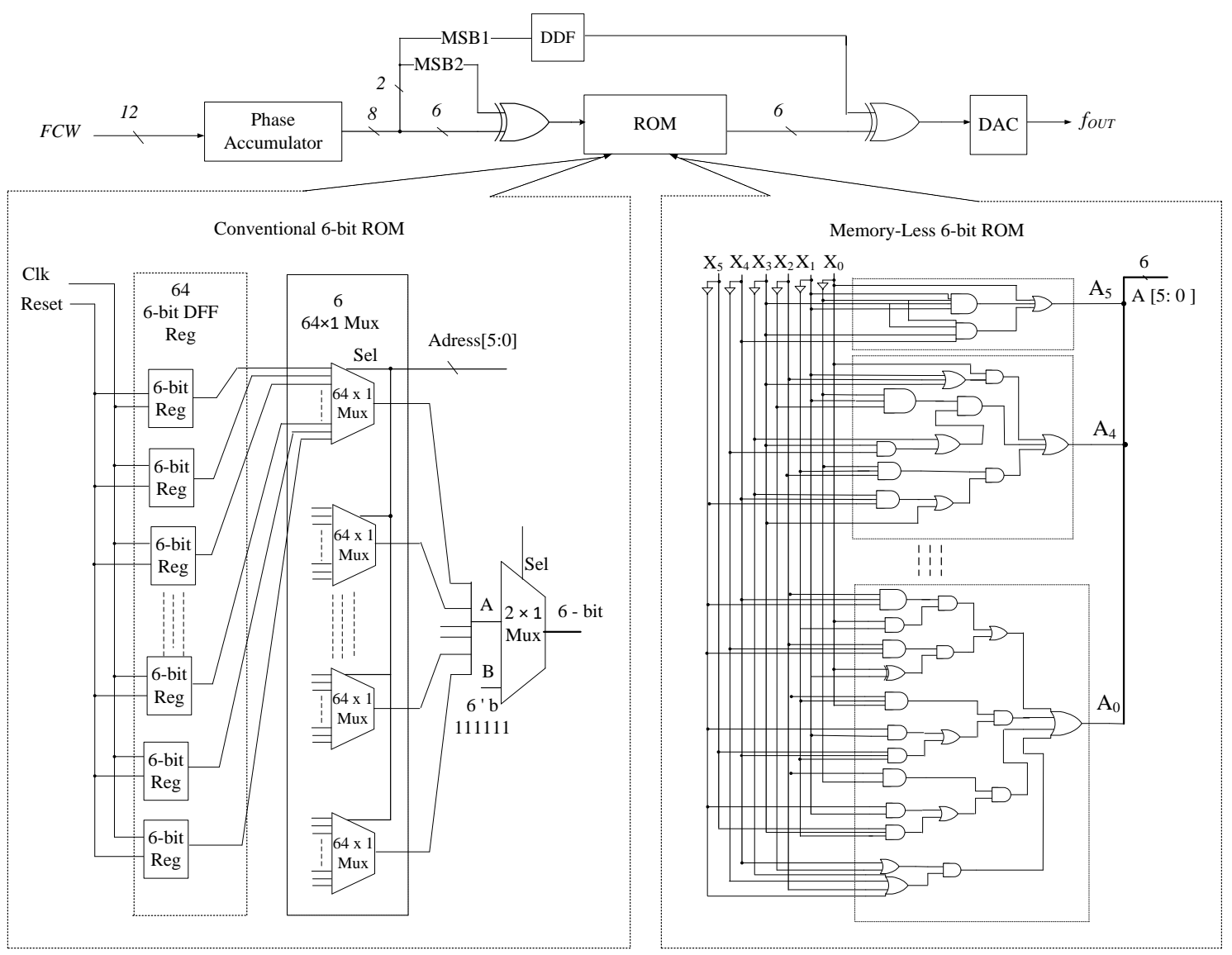

Figure 6. Conventional-memoryless 6-bit ROM circuit comparison

The 6-bit ROM requires 64 6-bit registers, six $64 \times 1$ multiplexers, and an extra $6 \times 1$ multiplexer to accomplish the sine amplitude waveform. The designed circuits of memory-less and conventional ROMs were synthesized in Synopsys software to obtain the optimized register transfer level (RTL) viewer, number of slices, and area dimension. Replacing the memory-less ROM circuit instead of conventional 6-bit ROM circuit reduces $56 \%$ of the lower area dimension and $32.6 \%$ of the number of cells. Table 2 shows the comparison of the number of cells, nets, and area dimension between the conventional ROM LUT and memory-less ROM. Comparison of the number of cells, nets, and area dimension of conventional and memory-less ROM.

Table 2. Comparison results of conventional and memory-less ROM logic gates

\begin{tabular}{cccc}
\hline (6-bit) ROM & No. Nets & No. Cells & Area $(\mu \mathrm{m} 2)$ \\
\hline Conventional & 98 & 106 & 2105.6112 \\
Memory-less & 66 & 72 & 924.739 \\
\hline
\end{tabular}

\section{RESULTS AND ANALYSIS}

The proposed DDFS system was programmed on the Cyclone III FPGA kit board and connected to the DAC hardware. The measured results of the frequency waveform and spectrum shown in Figures 7(a) and (b), respectively, are consistent with the expected results. The signal-to-noise ratio (SNR) is mathematically calculated using the following equation:

$$
S N R=6.02 M+1.76 d B \quad[20-22]
$$

As shown in (8), the $S N R$ value depends on the number DAC input bits (M). M=6 bits in this study; hence, $\mathrm{SNR}=37.88 \mathrm{~dB}$. The spectrum analyzer image shows that the measured DDFS output waveforms exhibit an SNR of approximately $45 \mathrm{~dB}$ as shown in Figure 7(b). 


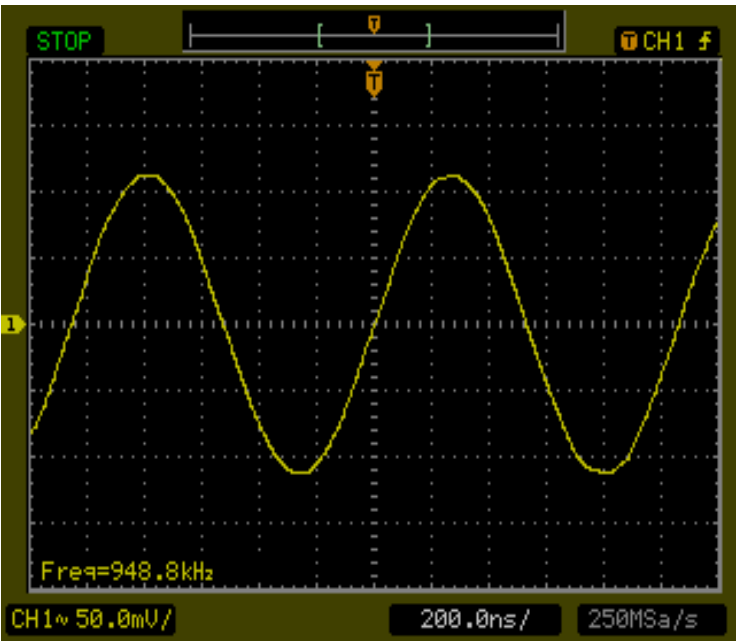

(a)

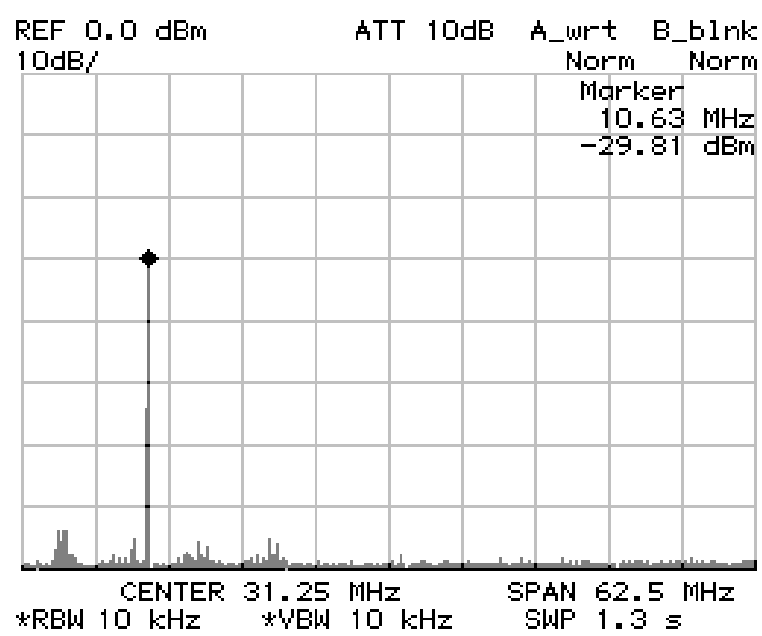

(b)

Figure 7. (a) Sine waveform of the DDFS, (b) Measured SNR waveform of DDFS output

Table 3 presents the comparison of the ROM size technique, spurious-free dynamic range (SFDR), and additional hardware used in the proposed design with several other parameters. The proposed design has no (DFF register) ROM bit size. The comparison between the proposed DDFS system and previous works is given in Table 2.

Table 3. Comparison results of conventional and memory-less ROM logic gates

\begin{tabular}{lllllll}
\hline Ref. & PA (bit) & Amp. & $\begin{array}{l}\text { ROM Size } \\
\text { (DFF (Reg.) }\end{array}$ & ROM Technique & Additional Hardware & SFDR (dB) \\
\hline$[4]$ & 11 & 9 & 368 & $\begin{array}{l}\text { Quad Line } \\
\text { Approximation Tech. }\end{array}$ & 5 Adders +1Mux + complementor & 55 \\
{$[5]$} & 9 & 6 & NA & $\begin{array}{l}\text { ROM-DAC } \\
\text { NA }\end{array}$ & 35 \\
{$[6]$} & 9 & 8 & NA & ROM-Less & $3: 8$ thermometer decoder & 42 \\
{$[7]$} & 9 & 8 & NA & ROM-less & NA & 44 \\
{$[23]$} & 8 & 6 & 112 & Quadrat Decompos. & 1 Adder + 1 Multiplier + (3:8) & 30 \\
& & & & Tech. & Decoder & 40.7 \\
{$[24]$} & 12 & 9 & 224 & 2 Splitted ROM & $3: 8$ thermometer decoder & 37.5 \\
{$[25]$} & 8 & 6 & NA & Nonlinear DAC & 139 Logic cells + 6-bit DFF & 45 \\
Proposed & 12 & 6 & 0 & Memory-Less ROM & Register & \\
\hline
\end{tabular}

\section{CONCLUSION}

Multiple types of parallel prefix adders used in pipelined PA designed circuits are coded using Verilog HDL code and verified in Quartus II software. The KS-based PA outperforms other conventional PA architectures. Therefore, KS-based PA is used in this research to improve the PA throughput. In addition to the quarter wave symmetry that reduces $75 \%$ of the ROM size, the memory-less circuits have been applied as a novel approach for ROM design instead of the conventional 6-bit ROM. This technique replaces $384 \mathrm{DFF}$ registers and multiplexers with 139 AND, OR, and XOR logic circuits, thereby reducing the lower area dimension and number of cells by $56 \%$ and $32.6 \%$, respectively. The proposed DDFS system is coded in Verilog HDL, successfully simulated and implemented with Cyclone III FPGA kit board, and connected to the DAC circuit for verification using waveform and spectrum analyzer devices. The measured results of the frequency waveform and spectrum analyzer are compared with the expected results. These performances provide the present DDFS design with further flexibility for applications in wireless communication systems.

\section{REFERENCES}

[1] S. H. Ibrahim, S. H. Ali and Mhd Shabiul Islam, "High Speed Direct Digital Frequency Synthesizer with Pipelining Phase Accumulator Based on Brent-Kung Adder," 10th IEEE International Conference on Semiconductor Electronics, pp. 547-550, 2012. 
[2] D. A. Sunderland, et al., "CMOS/SOS frequency synthesizer LSI circuit for spread spectrum communications," IEEE Journal of Solid-State Circuits, vol. 19, no. 4. pp. 497-506, 1984.

[3] H. Chen, J. Chen, Y. Li and Y. Wu., "A Quarter-ROM DDS Using Phase Accumulator with Subtraction," IEEE International Conference on Consumer Electronics-Taiwan (ICCE-TW), pp. 1-2, 2018.

[4] Y. Byung-Do, et al., "An 800-MHz low-power direct digital frequency synthesizer with an on-chip D/A converter," IEEE Journal of Solid-State Circuits, vol. 39, no. 5, pp. 761-774, 2004.

[5] G. Xueyang, F. F. Dai, J. D. Irwin, and R. C. Jaeger, "A 9-bit $2.9 \mathrm{GHz}$ direct digital synthesizer MMIC with direct digital frequency and phase modulations," IEEE Microwave Symposium Digest, pp. 1125-1128, 2009.

[6] M. Beheshti, and A. Jannesari, "A 2-GHz ROM-less direct digital frequency synthesizer based on an analog sinemapper circuit," 24th Iranian Conference on Electrical Engineering (ICEE), pp 1603-1608, 2016.

[7] W. Jun-Hong, Y. Ching-Yuan, and J. YI-LIN, "A low-power direct digital frequency synthesizer using an analogue-sine-conversion technique," IEEE Low Power Electronics and Design (ISLPED), pp. 193-197, 2011.

[8] A. Garg, D. Agrawal, P. Kularia at al, "Area efficient modified booth adder based on sklansky adder," 2nd International Conference for Convergence in Technology (I2CT), pp. 308-312, 2017.

[9] B. Tapasvi, et al., "Implementation of 64-bit Kogge Stone Carry Select Adder with ZFC for efficient area," IEEE International Conference on Electrical, Computer and Communication Technologies, pp. 1-6, 2015.

[10] B. Tapasvi, K. Bala Sinduri, B.G.S. Lakshmi, N. Udaya Kumar, "Implementation of 64-bit Kogge Stone Carry Select Adder with ZFC for efficient area," IEEE International Conference on Electrical, Computer and Communication Technologies (ICECCT), Coimbatore, India, Mar. 2015.

[11] S. Nikhil, P. Vijaya Lakshmi, "Implementation of a high speed multiplier desired for high-performance applications using kogge stone adder," International Conference on Inventive Computation Technologies (ICICT), Coimbatore, India, 26-27 Aug. 2016, 2016.

[12] Aradhanan Raju, Sudhir Kumar Sa, "Design and performance analysis of multipliers using Kogge Stone Adder," 3rd International Conference on Applied and Theoretical Computing and Communication Technology (iCATccT), Tumkur, India, 2017.

[13] N. U. Kumar, et al., "Implementation and comparison of VLSI architectures of 16-bit carry select adder using Brent Kung adder," Int. Conference on Innovations in Power and Advanced Computing Technologie, pp. 1-7, 2017

[14] A. Beaumont-Smith and L. Cheng-Chew, "Parallel prefix adder design," Computer Arithmetic, pp. 218-225, 2001.

[15] V. Shilpa Ameta, "Design and Analysis of 8-bit Carry Look Ahead Adder using TG and GDI Technology," International Journal of Innovative Research in Science, Engineering and Technology, vol. 6, no. 7, pp. 13848-13857, 2017.

[16] K. S. Shekhawat, and G. Sujediya, "Design and Analysis of RCA and CLA using CMOS, GDI, TG and ECRL Technology," International Journal of Advanced Engineering Research and Science, vol. 4, no. 11, pp. 126 -129, 2017.

[17] S. Alkurwy, et al., "A low power memoryless ROM design architecture for a direct digital frequency synthesizer," Turkish Journal of Electrical Engineering \& Computer Sciences, vol. 25, no. 5, pp. 4023-4032, 2017.

[18] S. Alkurwy, S. Ali, Mhd S. Islam, "Implementation of low power compressed ROM for direct digital requency synthesizer," International Conference on Semiconductor Electronics (ICSE2014), pp. 309-312, 2014.

[19] Salah Alkurwy, "A novel approach of multiplier design based on BCD decoder," Indonesian Journal of Electrical Engineering and Computer Science (IJEECS), vol. 14, no. 1, pp. 38-43, 2019.

[20] P. O'Leary, and Maloberti, F., "A direct-digital synthesizer with improved spectral performance," IEEE Transactions on Communications, vol. 37, no. 7, pp. 1046-1048, 1991.

[21] A. Torosyan and A. N. Willson, "Exact analysis of DDS spurs and SNR due to phase truncation and arbitrary phase-to-amplitude errors," Proce. of the IEEE International Frequency Control Symposium and Exposition, 2015.

[22] K. Gentile and R. Cushing, "A Technical Tutorial on Digital Signal Synthesis," Analog Devices, pp. 16-19, 1999. [Online]. Available: www.analog.com.

[23] A. Gutierrez-Aitken, et al., "Ultra high speed direct digital synthesizer using InP DHBT technology," Gallium Arsenide Integrated Circuit (GaAs IC) Symposium, pp. 265-268, 2001.

[24] S. E. Turner, R. T. Chan, and J. T. Feng, "ROM-Based Direct Digital Synthesizer at 24 GHz Clock Frequency in InP DHBT Technology," Microwave and Wireless Components Letters, vol. 18, no. 8, pp. 566-568, 2008.

[25] D. Wu, et al., "An ultra-high-speed direct digital synthesizer with nonlinear DAC and wave correction ROM," Analog Integrated Circuits and Signal Processing, vol. 73, no. 1, pp. 291-300, 2012.

\section{BIOGRAPHIES OF AUTHORS}

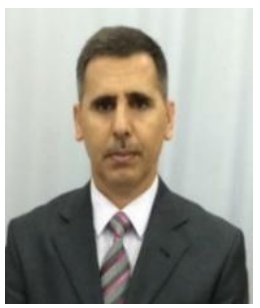

Salah H. Alkurwy received his BSc. Degree in electrical engineering, MSc degree in electrical and electronic engineering from University of Belgrade/Serbia. He received his $\mathrm{Ph} . \mathrm{D}$. degree in Electrical Electronic and System Engineering in 2015, from Universiti Kebangsaan Malaysia (UKM), Malaysia. He has published in the areas of Electronic, Frequency synthsizer and digital system. Presently, he is an Associate Professor and a member of the Department of Electronic Engineering, College of Engineering, University of Diyala / Iraq. His research Interest includes Digital circuits and system design, digital electronics, Microelectronic and HDL programming. 


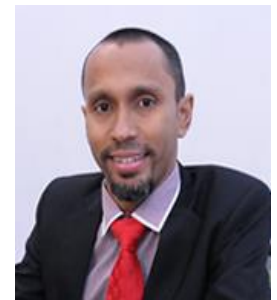

Sawal Hamid Md Ali received the M.Sc. and Ph.D. degrees from the University of Southampton, U.K., in 2004 and 2010, respectively. He is currently an Associate Professor with Universiti Kebangsaan Malaysia (UKM) and also holding a position of the Head of Programme for Electric \& Electronic, Department of Electric, Electronic and System Engineering, Faculty of Engineering and Built Environment, UKM. He has published in the areas of analog IC Design, VLSI and digital system. His current research interests include wearable systems, system-on-chip design, and pervasive computing.

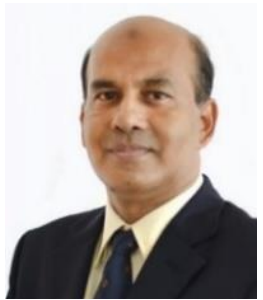

Md. Shabiul Islam received his B.Sc. from Department of Applied Physics \& Electronics, Rajshahi University, Bangladesh in 1985. M.Sc. degree in the area of Micro Controller Based System Design from Department of Electrical, Electronics \& System Engineering, Universiti Kebangsaan Malaysia (UKM), in 1997. He received his Ph.D. degree in VLSI design from Faculty of Engineering (FOE), Multimedia University (MMU), Malaysia in 2008. Currently, he is a professor at Multimedia University (MMU), Cyberjaya, Malaysia. His current interests are in energy harvesting related circuit, VLSI, digital design \& embedded system

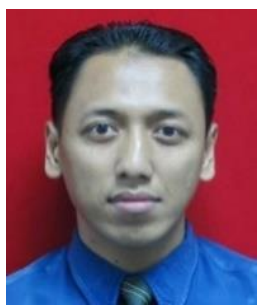

Mohd Faizul Mohd Idros received the BSc in Electronics 2002, MSc. Degree in Microelectronics and $\mathrm{Ph} . \mathrm{D}$. degree from Department of Electrical, Electronics \& System Engineering, Universiti Kebangsaan Malaysia (UKM), Malaysia in 2006 and 2015, respectively. Currently, he is a Lecturer at Electronic Engineering Studies, Universiti Teknologi MARA. He has published in the areas of IC Design, VLSI and digital system. His current interests are in System design, IC Design, Sensor, VLSI, digital design and embedded system. 